\title{
Application of Fourier-Padé Approximation in Analysis of Holographic Photonic Crystal Structures
}

\author{
D. Sevic*, S. Savic-Sevic, D. Pantelic, B. Jelenkovic and B.P. Marinkovic \\ Institute of Physics, Belgrade, Serbia
}

\begin{abstract}
In this paper a method based on the Fourier transform and Padé approximants is investigated for analysis of holographic photonic crystal structures fabricated in Laboratory for Photonics, Institute of Physics, Belgrade. Padé approximants are numerical tool that can be used to accelerate the convergence of a slowly converging sequence.
\end{abstract}

PACS numbers: 42.40.Eq, 42.70.Qs. $02.30 . \mathrm{Nw}$

\section{Introduction}

Photonic crystal structures are fabricated in Laboratory for Photonics, Institute of Physics, Belgrade, by the holographic technique [1]. Fast Padé approximation is used as a numerical aid in studies of photonic crystals, see recent publication [2] and some of references therein.

In this paper a method based on the Fourier transform and Padé approximants is investigated for analysis of holographic photonic crystal structures. The Padé approximants are numerical tool that can be used to accelerate the convergence of a slowly converging sequence. For a power series defined as:

$$
F_{n}(z)=\sum_{n=0}^{N} c_{k} z^{k}
$$

of order $N$ in the variable $z$ (real or complex) with coefficients $c_{k}$, Padé approximant is a rational fraction $P_{m}(z) / Q_{n}(z)$, which approximates the fully converged values of the function $F_{n}(z)$. If we let $z=\mathrm{e}^{-\mathrm{j} k n}$, then Eq. (1) corresponds to discrete Fourier transform (DFT), so the Padé method is used to improve spectral resolution of fast Fourier transform (FFT), the most popular tool in spectral analysis. This method is usually called Fourier-Padé (FP) approximation.

We applied Fourier-Padé approximation to analyze photonics crystal structures fabricated in our laboratory, enhancing the FFT analysis of the investigated samples. Our FP is implemented as a C code.

\section{Analyzed samples}

One-dimensional photonic crystals (PC) are fabricated in a dichromate-sensitized pullulan (DCP) by holography. Photonic crystals are created as volume reflection holograms $[3,4]$. A single-frequency, diode pumped

\footnotetext{
* corresponding author; e-mail: sevic@ipb.ac.rs
}

Nd-YAG laser, at $532 \mathrm{~nm}$, is used for exposure. Band gaps in the visible range are observed. In addition to the volume grating, a surface relief grating is also formed. In this paper we will analyze this surface relief structure of one dimensional PC. Surface relief of one of the samples measured by the profilometer is shown in Fig. 1. Digital image of a DCP hologram obtained by the optical microscope is shown in Fig. 2.

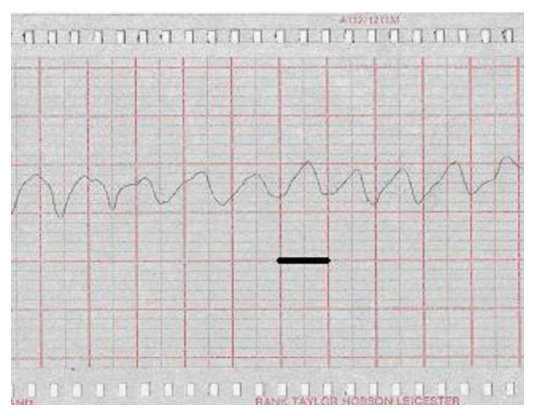

Fig. 1. Surface relief of one of the DCP holograms measured by the profilometer. Bar corresponds to $50 \mu \mathrm{m}$.

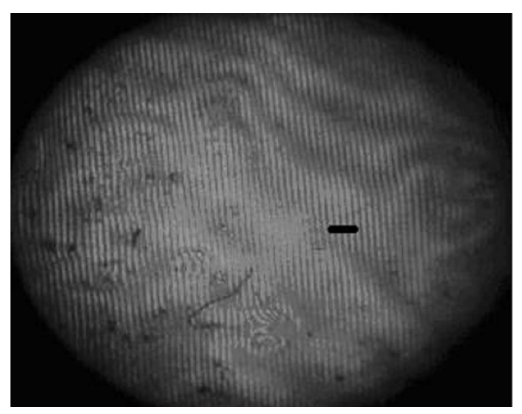

Fig. 2. Digital image of a DCP hologram obtained by optical microscope. Bar corresponds to $20 \mu \mathrm{m}$. 


\section{Analysis of photonic crystal DCP structures using FP}

The Fourier transform of the surface relief shown in Fig. 1 is calculated and presented in Fig. 3. FP of the same data is calculated and presented in Fig. 4. Spatial frequency is in the range 0 to $\pi$ and is scaled to 1024 points. Spectral component, easily visible in Fig. 4, is indiscernible on the Fourier transform spectrum, Fig. 3, masked by spectral leaking. FP provides enhanced insight into spatial frequency characteristics of PC structures.

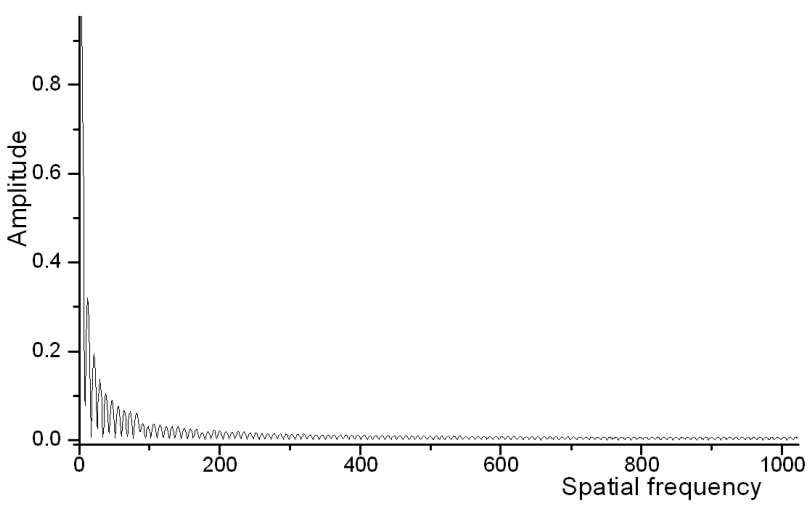

Fig. 3. Fourier transform of surface relief shown in Fig. 1.

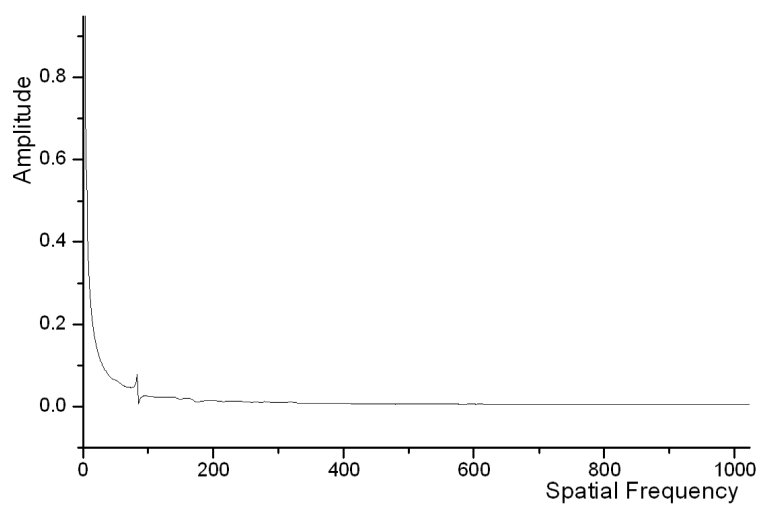

Fig. 4. Fourier-Padé approximation of surface relief shown in Fig. 1.

Unlike the 2D Fourier transform, 2D Padé approximation is not separable, so calculating the $2 \mathrm{D} \mathrm{FP}$ is very demanding task $[5,6]$. Fortunately, PC structures which are of interest here have very directional structure, so we will use Padé approximation to enhance FFT analysis only in the direction of interest, which is horizontal for the grating shown in Fig. 2.

Region of interest for analysis of the digital image of a DCP hologram obtained by optical microscope is shown in Fig. 5a.

The Fourier transform of the image (a) is shown in Fig. 5b, and Fourier Padé approximation of the same image is shown in Fig. 5c. Output is reordered so the

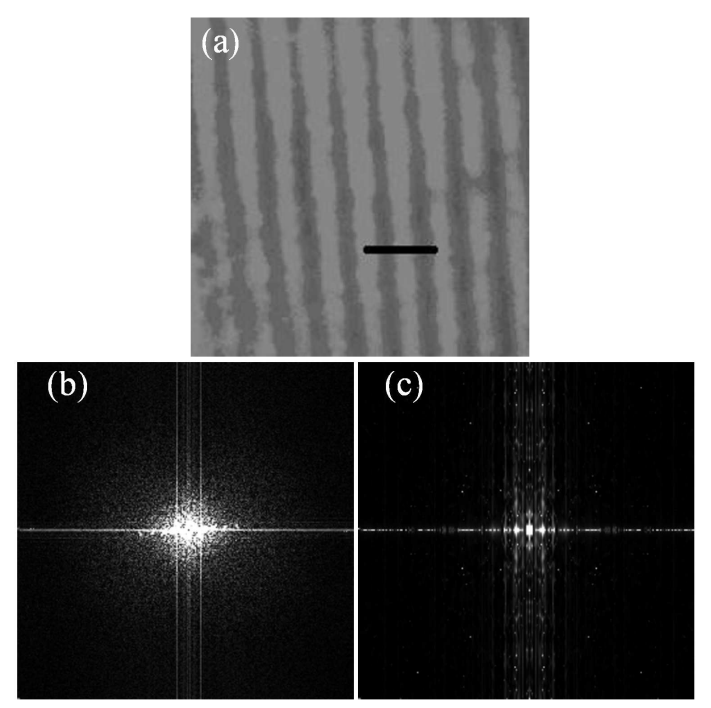

Fig. 5. (a) Analyzed part of the image shown in Fig. 2 (bar corresponds to $100 \mu \mathrm{m}$ ). (b) Fourier transform of the (a), (c) Fourier-Padé approximation of the (a), enhanced in the horizontal direction.

DC component is in the centre. Similar to the 1D case shown in Fig. 3, blurring of the Fourier transform spectrum is obvious in Fig. 5b, better spectral component localization is easy to notice in the Fig. 5c.

\section{Conclusion}

In this paper a method based on Fourier transform and Padé approximants is investigated for analysis of holographic photonic crystal structures fabricated in our laboratory. Compared to usual use of the Fourier transform, structural analysis of fabricated samples is enhanced by better spatial frequency resolution provided by Fourier-Padé approximation.

\section{Acknowledgments}

The work has been done within the projects OI 141011 and OI 141003 financed by the Ministry of Science and Technological Development, Republic of Serbia.

\section{References}

[1] S. Savic-Sevic, D. Pantelic, R. Gajic, G. Isic, Acta Phys. Pol. A 112, 1079 (2007).

[2] Y. Zhang, W. Zheng, M. Xing, G. Ren, H. Wang, L. Chen, Opt. Commun. 281, 2774 (2008).

[3] Z. Ye, J. Zheng, D. Liu, S. Pei, Phys. Lett. A 299, 313 (2002).

[4] R. Ma, J. Xu, W.Y. Tam, Appl. Phys. Lett. 89, 081116 (2006).

[5] M.F. Calaghan, D.J. Larkman, J.V. Hajnal, Magn. Res. Med. 54, 1490 (2005).

[6] Dz. Belkic, Nucl. Instrum. Methods Phys. Res. A 471, 165 (2001). 